\title{
The generation of disinhibition bursts of dopaminergic neurons in the basal ganglia
}

\author{
Collin J Lobb*, Carlos A Paladini, Charles J Wilson, Todd W Troyer \\ From Nineteenth Annual Computational Neuroscience Meeting: CNS*2010 \\ San Antonio, TX, USA. 24-30 July 2010
}

The majority of the synapses onto dopaminergic neurons in the substantia nigra pars compacta (SNpc) are GABAergic and originate from spontaneously active neurons in the substantia nigra pars reticulata ( $\mathrm{SNpr}$ ) and globus pallidus (GP) [1]. This tonic $\mathrm{GABA}_{\mathrm{A}}$-mediated inhibition [2] is opposed by tonic NMDA-mediated excitation from the subthalamic nucleus (STN) [3], suggesting that dopaminergic neurons may be in a high conductance state [4]. Thus, in vivo we expect SNpc activity to depend on the neuron's intrinsic pacemaking currents acting in tandem with tonic NMDA and $\mathrm{GABA}_{\mathrm{A}}$-mediated synaptic currents.

We first investigated the high conductance state in a coupled-oscillator model of the SNpc dopaminergic neuron [5]. This neuron model is capable of producing bursts through phasic activation of NMDA receptors, but strong excitation can prevent firing due to inactivation block. However, by adding $\mathrm{GABA}_{\mathrm{A}}$ receptors $\left(\mathrm{E}_{\mathrm{GABA}}=-60 \mathrm{mV}\right)$, we found that in the high conductance state the model is capable of firing single spikes. This was parametrically explored using a range of constant NMDA and $\mathrm{GABA}_{\mathrm{A}}$ conductances.

The likelihood of strong GABAergic tone in vivo raises the possibility that phasic disinhibition may be an alternative mechanism to phasic excitation for triggering reward-related bursts of action potentials [6]. To investigate the possible dynamics of disinhibition and how it may cause bursting, we used a modified version of an integrate-and-fire based model of the basal ganglia [7]. A $\mathrm{SNpc}$ nucleus receiving afferent inputs from the striatum, GP, STN, and SNpr were added to the network model. We captured the spike input to a random SNpc dopaminergic neuron in the network model and used these spike trains to generate synaptic input to the conductancebased coupled oscillator model of the dopaminergic

\footnotetext{
* Correspondence: collin.lobb@utsa.edu

Neurosciences Institute, University of Texas at San Antonio, San Antonio, TX 78249, USA
}

neuron. Phasic activation of the D1-expressing medium spiny neurons in the striatum (D1STR) produced disinhibition bursts in dopaminergic neurons through the direct pathway (D1STR to SNpr to SNpc).

It has previously been shown that direct pathway medium spiny neurons have collaterals that terminate in the GP [8]. This connection was added to the network model (D1STR to GP). We found that striatal activation of the indirect pathway (D1STR-GP-STN-SNpr) through this connection increased the disinhibition burst frequency.

These studies suggest that striatal activation is a robust means by which disinhibition bursts can be generated by SNpc dopaminergic neurons, and that the indirect pathway may enhance disinhibition bursting.

Published: 20 July 2010

\section{References}

1. Bolam JP, Smith $Y$ : The GABA and substance $P$ input to dopaminergic neurones in the substantia nigra of the rat. Brain Res 1990, 529(12):57-78.

2. Paladini CA, Celada P, Tepper JM: Striatal, pallidal, and pars reticulata evoked inhibition of nigrostriatal dopaminergic neurons is mediated by GABA(A) receptors in vivo. Neuroscience 1999, 89(3):799-812.

3. Chergui K, Akaoka H, Charlety PJ, Saunier CF, Buda M, Chouvet G: Subthalamic nucleus modulates burst firing in nigral dopamine neurones via NMDA receptors. Neuroreport 1994, 5(10):1185-1188.

4. Destexhe A, Rudolph M, Paré D: The high-conductance state of neocortical neurons in vivo. Nat Rev Neurosci 2003, 4(9):739-751.

5. Kuznetsov AS, Kopell NJ, Wilson CJ: Transient high-frequency firing in a coupled-oscillator model of the mesencephalic dopaminergic neuron. J Neurophysiol 2006, 95(2):932-947.

6. Schultz W: Predictive reward signal of dopamine neurons. J Neurophysiol 1998, 80(1):1-27.

7. Humphries MD, Steward RD, Gurney KN: A physiologically plausible model of action selection and oscillatory activity in the basal ganglia. J Neurosci 2006, 26(50):12921-12942.

8. Kawaguchi Y, Wilson CJ, Emson PC: Projection subtypes of rat neostriatal matrix cells revealed by intracellular injection of biocytin. J Neurosci 1990, 10(10):3421-38.

doi:10.1186/1471-2202-11-S1-P112

Cite this article as: Lobb et al:: The generation of disinhibition bursts of dopaminergic neurons in the basal ganglia. BMC Neuroscience 2010 11(Suppl 1):P112. 